\title{
European Vitreoretinal Society Macular Hole Study, Prognostic Factors for Anatomical and Functional Success
}

\author{
Barbara Parolini ${ }^{1}$, Zofia Michalewska ${ }^{2}$, Joshua S Hardin ${ }^{3}$, Didier Ducournau ${ }^{4}$, Ihab Saad Othman ${ }^{5}$, Mohammed F. Faramawi ${ }^{6}$, \\ Ron A. Adelman ${ }^{7}$, Ahmed B. Sallam ${ }^{3}$ \\ ${ }^{1}$ Istituto Clinico S.Anna di Brescia, Italy \\ 2 Ophthalmic Clinic "Jasne Blonia", Rojna, Poland \\ ${ }^{3}$ Jones Eye Institute, University of Arkansas for Medical Sciences, Little Rock, Arkansas, USA \\ ${ }^{4}$ Clinique Sourdille, Nantes, France. \\ ${ }^{5}$ Ophthalmology Department, Cairo University, Egypt \\ ${ }^{6}$ Epidemiology Department, College of Public Health and Biomedical informatics, College of Medicine, University of Arkansas for Medical \\ Sciences, Little Rock, Arkansas, USA \\ ${ }^{7}$ Ophthalmology Department, Yale University School of Medicine, New Haven, CT, USA
}

Epub: June 20, 2020

\begin{abstract}
Background: To identify prognostic preoperative and intraoperative factors for anatomical and visual success of idiopathic macular hole $(\mathrm{MH})$ surgery.

Methods: We conducted a non-randomized, collaborative multicenter study using data of $4207 \mathrm{MH}$ surgery from 140 surgeons. Main study outcomes were anatomical closure and best corrected visual acuity (BCVA) improvement postoperative at 6-12 months.

Results: Information on anatomical success was available for 4138 eyes of 4207 operations. Anatomical closure of $\mathrm{MH}$ was achieved in $85.7 \%$ ( 3546 eyes). Closure was higher in smaller MH (stages 1-2 versus stage 3: OR=0.35; stage 2 versus stage 4: $\mathrm{OR}=0.16$, and in $\mathrm{MH}$ with shorter duration before the operation ( $\mathrm{OR}=0.94)$. Macular Holes were more likely to close when dyes were used to facilitate internal limiting membrane (ILM) peeling (odds ratio=1.73 to 3.58 ). The most important predictors of postoperative BCVA were the preoperative BCVA (estimate $=0.39, p<0.001$ ) and closure of the macular hole (estimate $=0.34$, $p<0.001$ ). We observed Larger improvement in BCVA in combined vitrectomy and phacoemulsification (estimate $=0.10$ ) and post cataract surgery in phakic eyes (estimate $=0.05)$. Retinal tears occurred in $5.1 \%$ of eyes, and were less with use of trocars (OR= 1.246) and in combined vitrectomy/ phacoemulsification surgery $(O R=-0.688)$.

Conclusion: This international survey confirmed that staining with dyes improves anatomical results but not visual outcomes. After surgery, visual acuity improved during the first year, and final visual acuity was better in both pseudophakic eyes and eyes that underwent cataract surgery during the first year following $\mathrm{MH}$ repair.
\end{abstract}

\section{KEY WORDS}

Macular Hole; Vitrectomy; Inverted ILM Flap; Full-Thickness Macular Hole; ILM Peeling; Complete Vitrectomy; Combined Surgery; Positioning; Internal Limiting Membrane.

Copyright (C 2020, Med Hypothesis Discov Innov Ophthalmol. This is an open-access article distributed under the terms of the Creative Commons Attribution-NonCommercial 4.0 International License (http://creativecommons.org/licenses/by-nc/4.0/) which permits copy and redistribute the material just in noncommercial usages, provided the original work is properly cited.

Correspondence to: Ahmed B. Sallam, MD, PhD; FRCOphth, Jones Eye Institute, University of Arkansas for Medical Sciences, Little Rock, AR 72211. Phone: 501-526-6000 x 1225; fax: 501-603-1289. E-mail: ahmedsallam11@yahoo.com

How to cite this article: Parolini B, Michalewska Z, Hardin JS, Ducournau D, Othman IS, Faramawi MF, Adelman RA, Sallam AB, European Vitreoretinal Society Macular Hole Study, Prognostic Factors for Anatomical and Functional Success. Med Hypothesis Discov Innov Ophthalmol. 2020; 9(3): 198-207. 


\section{INTRODUCTION}

Idiopathic macular hole represents a common vitreoretinal pathology with an estimated population-incidence of approximately 9 cases annually [1]. While non-surgical modalities including intravitreal injection of ocriplasmin or gas are available for the treatment of macular hole (MH) [2, 3], surgical treatment with pars plana vitrectomy (PPV) has remained the main standard approach. Pars plana vitrectomy in MH surgery was first reported by Kelly and Wendel in 1991 [4]. Their initial success rate was $58 \%$. Since then, surgical techniques have been refined and results from randomized controlled trials $[5,6]$ and contemporary real world studies reported an anatomical closure rate of $85 \%$ or more, and visual improvement of approximately 2-3 Snellen line [7-10].

Large macular holes are associated with worse visual prognosis and postoperative photoreceptor status has a confirmed correlation with visual acuity $[11,12]$. However, there is paucity of evidence regarding the influence of intraoperative details on the results of MH surgery. Specifically, the role of intraocular tamponades [10, 13, 14], internal limiting membrane (ILM) peeling [15-20], ILM staining and the influence of the types of staining dye [21], and postoperative positioning [22-29] are still frequently debated. The main conclusion derived from a review of the literature is that metaanalyses and well-conducted, controlled trials are necessary to determine which surgical details are prognostic in $\mathrm{MH}$ surgery $[30,31]$. Given the differences in the reporting outcomes in each study, and the varying surgical details, a meta-analysis would hardly be possible. A randomized clinical trial on a sufficiently large group of patients would also be costly, time consuming and may not reflect routine clinical care.

The European VitreoRetinal Society (EVRS) has previously conducted large studies examining the treatment of retinal detachment and macular edema in the real-world setting [3237]. In the current investigation, a total of 4,207 operations for idiopathic $\mathrm{MH}$ performed by 140 surgeons from 28 countries were evaluated.

We aimed to report anatomical and functional outcomes of surgery for $\mathrm{MH}$ and to analyze the influence of different pre-, intra-operative factors. While one larger study has reviewed reoperation and retinal detachment with $\mathrm{MH}$ surgery [20], to our knowledge this is the largest comprehensive report on the anatomical and visual outcomes of $\mathrm{MH}$ in the literature to date.

METHODS

The EVRS Macular Hole Study is a non-randomized, multicenter retrospective survey designed to identify pre-, intra-, and post-operative factors determining anatomical and functional success of $\mathrm{MH}$ repair. Macular hole operations reported between June 2008 and June 2012 by the society members were included. In total, 4,207 macular holes were operated by 140 vitreoretinal specialists from 28 countries across 4 continents (Figure 1 and 2 represent surgeons and eyes break down by country). The data gathered included lens status, hole duration, gauge, the use of trocars, suturing of scleral wounds, combined procedures, posterior hyaloid removal, internal limiting membrane (ILM) staining, ILM peeling, type of tamponade, completeness of tamponade, details of postoperative positioning, cataract formation, and complications including retinal tear and detachment (Table 1). The anatomical outcomes of repair were classified as follows; macular hole closed with edges not visible; hole open with edges visible and attached hole open with edges elevated; hole open with edges visible and attached. Only cases with macular hole closed and edges not visible were accounted as anatomical successes. Follow-up periods were 3-6 months, 612 months and $12+(12-15)$ months after surgery.

To identify the most relevant predictors for best corrected visual acuity (BCVA) at three postoperative follow-up periods, a linear mixed effects model with two nested random effects was considered [38]. The model involved considering a surgeon's (or group of surgeons') random effect to account for surgeon heterogeneity and a patient's random effect nested within the surgeon's random effect to account for patient heterogeneity.

The considered potential predictors for BCVA were preoperative BCVA at different follow-up visits, stage, size (small, medium, large), hole duration, lens status, anatomical success, vitrectomy completeness (core, subtotal, complete), gauge, trocar, suturing of scleral wounds, combined phacoemulsification, type of dye, extent of posterior hyaloid removal (limited to the posterior pole or large), quality of tamponade, type of tamponade (complete versus incomplete), concentration of gas, quality of postoperative positioning (either strict prone positioning or patients advised not to look up, or no positioning at all), and duration of postoperative positioning. The model was estimated by the maximum likelihood method through the Ime function in $R$ package [39] nlme [40]. Please define yellow colors

The predictors of the probability of anatomical success were evaluated through a multivariate logistic model with a random intercept to account for surgeon heterogeneity. The considered potential predictors for anatomical success were $\mathrm{MH}$ stage, hole duration, crystalline lens status, vitrectomy completeness, combined phacoemulsification, type of dye to stain ILM, posterior hyaloid removal, quality of tamponade, type of tamponade, concentration of gas, quality of postoperative positioning and duration of postoperative positioning. The 
model was estimated by the maximum likelihood method through $R$ package glmmML [41]. For all multivariate

regression analyses, model selection was based on the stepwise method with Akaike Information Criterion [42].

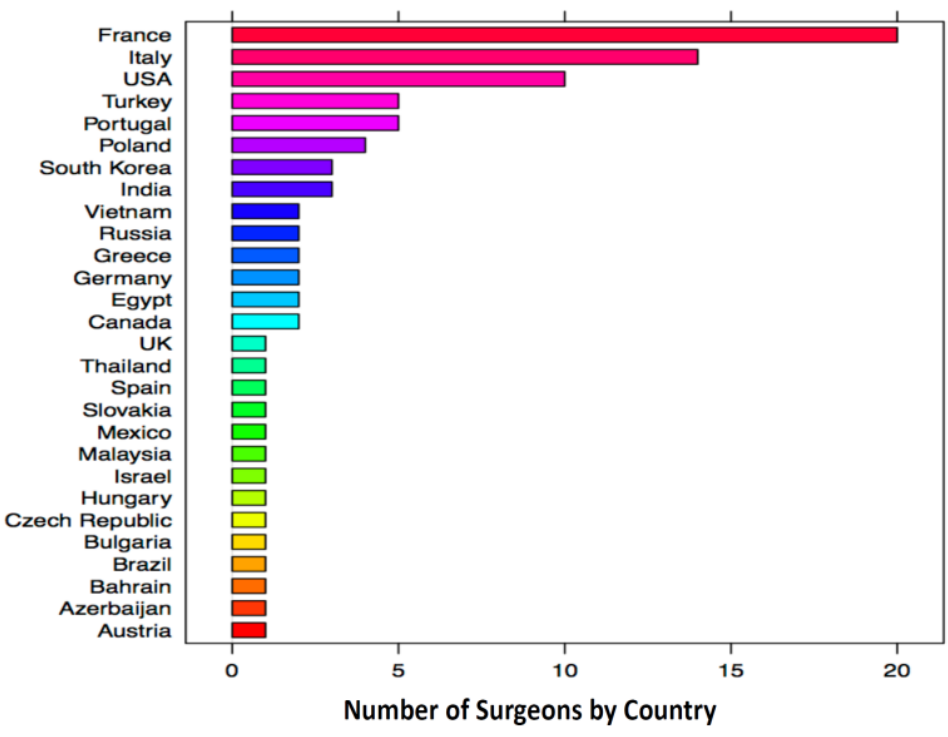

Figure 1. Number of Surgeons by Country.

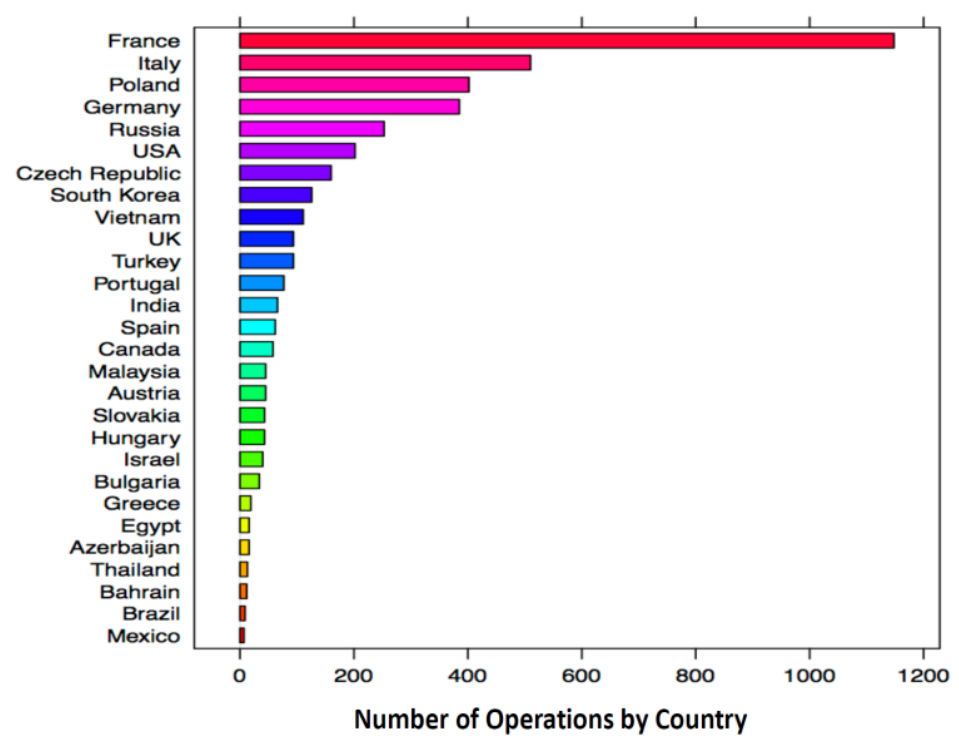

Figure 2. Number of Operations by Country. 
Table 1. Variables That Surgeons Were Asked to Report to Assess Anatomical and Visual Outcome of MH Surgery

\begin{tabular}{|c|c|c|c|c|c|}
\hline Clinical Findings & $\begin{array}{l}\text { Pars Plana } \\
\text { Vitrectomy }\end{array}$ & $\begin{array}{l}\text { Additional } \\
\text { Intraoperative details }\end{array}$ & Tamponade & $\begin{array}{l}\text { Anatomical and Visual } \\
\text { Outcomes }\end{array}$ & $\begin{array}{l}\text { Postoperative } \\
\text { Complications }\end{array}$ \\
\hline $\begin{array}{l}\text { - Stage of FTMH } \\
\text { - FMTH duration } \\
\text { - Pre-operative } \\
\text { BCVA }\end{array}$ & $\begin{array}{l}\text { - Date of operation } \\
\text { - Gauge } \\
\text { - Trocar use } \\
\text { - Suturing of scleral } \\
\text { wounds } \\
\text { - Combined } \\
\text { phacoemulsificatio } \\
\text { n vitrectomy } \\
\text { - Special techniques } \\
\text { - Intraoperative } \\
\text { complications }\end{array}$ & $\begin{array}{l}\text { - ILM peeling (yes/ no) } \\
\text { - Dye used (none, } \\
\text { trypan blue/ } \\
\text { membrane blue, } \\
\text { brilliant blue, } \\
\text { Indocyanine green, } \\
\text { other) } \\
\text { - Peeling (100\% sure/ } \\
\text { not sure) } \\
\text { - Technique of peeling } \\
\text { - Complications of } \\
\text { operation }\end{array}$ & $\begin{array}{l}\text { - Type of tamponade (air, SF6, } \\
\text { C2F6, C3F8, silicone oil) } \\
\text { - Complete ( } \geq 80 \% \text { ) fill/ } \\
\text { Incomplete tamponade } \\
\text { - Special techniques } \\
\text { - Postoperative positioning } \\
\text { (none, just look down, prone } \\
\text { positioning) } \\
\text { - Duration of postoperative } \\
\text { positioning }\end{array}$ & $\begin{array}{l}\text { - } \text { FTMH closed (edges } \\
\text { not visible) / flat } \\
\text { edges/elevated edges } \\
\text { - BCVA 3-6 months } \\
\text { - BCVA 6-12 months } \\
\text { - BCVA 12-15+ (12+) } \\
\text { months }\end{array}$ & $\begin{array}{l}\text { - Cataract } \\
\text { - Retinal tear } \\
\text { - Retinal } \\
\text { detachment } \\
\text { - MH } \\
\text { reoperation } \\
\text { - Hypotony }\end{array}$ \\
\hline
\end{tabular}

FTMH: full thickness macular hole; BCVA: best corrected visual acuity; ILM: internal limiting membrane; MH: macular hole

Table 2. Odds Ratios With 95\% Confidence Intervals (CI) in Parentheses and P-Values for the Regression Coefficients of the Selected Logistic Mixed Effects Model for the Probability of Anatomical Closure of Macular Hole.

\begin{tabular}{|l|l|l|}
\hline \multicolumn{2}{|l|}{ Odds ratio $(95 \% \mathrm{Cl})$} & p-value \\
\hline Stage & $0.35(0.20,0.61)$ & $<0.001$ \\
\hline Stage 3 FTMH & $0.16(0.09,0.28)$ & $<0.001$ \\
\hline Stage 4 FTMH & $0.94(0.91,0.97) \times 10^{-3}$ & $<0.001$ \\
\hline FTMH duration & & \\
\hline Type of Dye & $1.73(1.05,2.86)$ & $\mathbf{0 . 0 3 1}$ \\
\hline Brilliant blue & $2.51(1.34,4.69)$ & $\mathbf{0 . 0 0 4}$ \\
\hline Indocyanine green & $2.27(1.12,4.60)$ & $\mathbf{0 . 0 2 3}$ \\
\hline Trypan/Membrane blue & $3.58(1.31,9.75)$ & $\mathbf{0 . 0 1 3}$ \\
\hline Other & & \\
\hline
\end{tabular}

FTMH: full thickness macular hole; $\mathrm{P}<0.05$ in bold.

Table 3. Estimates With $95 \%$ Confidence Intervals (CI) in Parentheses and P-Values for the Regression Coefficients of the Selected Linear Mixed Effects Model for BCVA Variation in Macular Hole

\begin{tabular}{|l|l|l|}
\hline Coefficients & Estimate $(95 \% \mathrm{CI})$ & \multicolumn{1}{c|}{$\mathbf{p}$-value } \\
\hline Preoperative BCVA & $0.39(0.35,0.43)$ & $<\mathbf{0 0 1}$ \\
\hline $\mathbf{6 - 1 2}$ months BCVA & $0.09(0.08,0.10)$ & $<.001$ \\
\hline 12+ months BCVA & $0.11(0.10,0.13)$ & $<\mathbf{0 . 0 0 1}$ \\
\hline MH closed & $0.34(0.31,0.38)$ & $<\mathbf{0 . 0 0 1}$ \\
\hline Stage 3 MH & $-0.06(-0.09,-0.02)$ & $\mathbf{0 . 0 0 2}$ \\
\hline MH duration & $-0.11(-0.15,-0.07)$ & $<\mathbf{0 . 0 0 1}$ \\
\hline Incomplete intraocular tamponade & $-0.78(-1.16,-0.41) \times 10^{-3}$ & $<\mathbf{0 0 1}$ \\
\hline Pseudophakia & $0.05(0.00,0.10)$ & 0.075 \\
\hline Combined phacoemulsification, lens implantation and vitrectomy & $0.05(0.02,0.09)$ & $\mathbf{0 . 0 0 1}$ \\
\hline Duration of postoperative positioning & $0.10(0.06,0.14)$ & $<.001$ \\
\hline
\end{tabular}

BCVA: best corrected visual acuity; $\mathrm{MH}$ : macular hole; $\mathrm{P}<0.05$ in bold.

Table 4. Odds Ratios With 95\% Confidence Intervals (CI) in Parentheses and P-Values for the Regression Coefficients of The Selected Logistic Mixed Effects Model for the Probability of Successive Cataract Surgery following Macular Hole Surgery. $P<0.05$ in bold.

\begin{tabular}{|l|l|l|}
\hline Coefficients & Odds ratio $(95 \% \mathrm{CI})$ & $\mathrm{p}$-value \\
\hline Transconjunctival vitrectomy & $0.43(0.26,0.72)$ & $\mathbf{0 . 0 0 1}$ \\
\hline Gas dilution & $1.18(1.02,1.36) \times 10^{-1}$ & $\mathbf{0 . 0 2 3}$ \\
\hline C3F8 gas versus C2F6 gas & $2.02(1.04,3.92)$ & $\mathbf{0 . 0 3 8}$ \\
\hline SF6 gas versus C2F6 gas & $1.42(0.75,2.68)$ & 0.277 \\
\hline
\end{tabular}




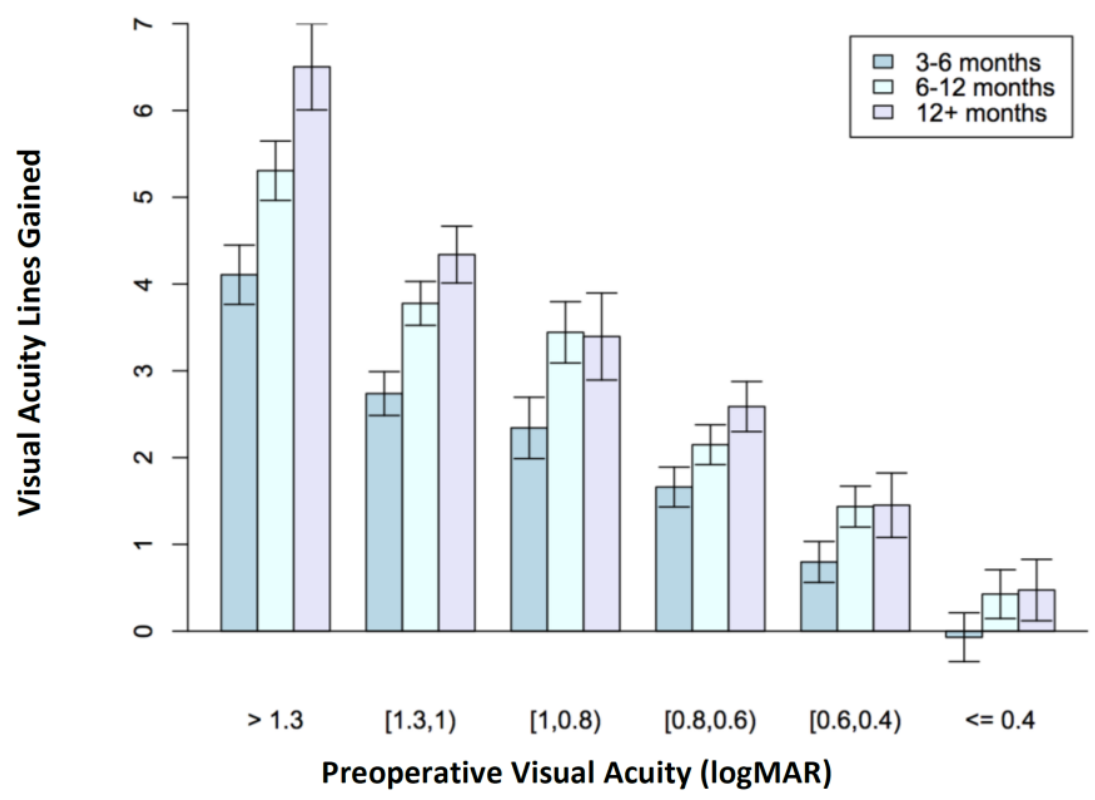

Figure 3. Postoperative Visual Acuity Lines Gained as a Function of Preoperative Vision in Logarithm of the Minimum Angle of Resolution (LogMAR) With 95\% Confidence Intervals for Average Levels. 3-6 months: 3-6 months Postoperation; 6-12 months: 6-12 months Postoperation; 12 ${ }^{+}$months: $12-15$ Months Postoperation.

\section{RESULTS}

\section{Preoperative characteristics}

Information on stage of $\mathrm{MH}$ was available for 3633 of 4207 (86.4\%) eyes. Stage $1 \mathrm{MH}$ was observed in 149 eyes $(4.1 \%$ of cases with information about stage), stage 2 in 661 eyes (18.2\%), stage 3 in 1294 eyes (35.6\%) and stage 4 in 1529 eyes (42.1\%). Stages 1 and 2 were merged since the frequency of stage 1 holes was considered low for reliable statistical analysis. Information about lens status was available for all but seven eyes. At the time of surgery, $0.4 \%$ (15 eyes) of patients were aphakic, $26.2 \%(1,100$ eyes) were pseudophakic and $73.5 \%$ (3,085 eyes) phakic. Combined phacoemulsification, lens implantation and vitrectomy surgery were performed in $25 \%$ of initially phakic eyes.

\section{Anatomical Results}

Information on anatomical success was available for 4138 of 4207 eyes. Overall anatomical success rate, defined as complete closure with edges not visible, was $85.7 \%$ (3546 eyes). Anatomical failure, with flat open $\mathrm{MH}$ was observed in $7.7 \%$ (318 eyes) of cases, and with elevated edges in $6.6 \%$ (274 eyes). The selected logistic mixed model for anatomical success is summarized in Table 2. Anatomical success was higher in smaller macular holes (stages 1-2 versus stage 3 : odds ratio $=0.35, p=0.001$; stage 2 versus stage 4: odds ratio $=0.16, p<0.001$ ) and in macular holes with shorter durations before the operation (odds ratio $=0.94, p<0.001$ ). When eyes with stage 2 macular holes were analyzed separately, the results were similar to those of the group including stages 1 and 2. Macular Holes were more likely to close when dyes were used to facilitate ILM peeling (odds ratio $=1.73$ to 3.58 ), compared to without the use of dyes; however, there were no statistically significant differences between various types of dyes (Indocyanine Green, Trypan/Membrane blue and Brilliant blue).

\section{Visual Acuity Results}

Figure 3 displays the visual acuity lines gained as a function of preoperative Logarithm of the Minimum Angle of Resolution (LogMAR) VA. Improvement in postoperative BCVA was statistically significant at all successive followup intervals (3-6 months versus 6-12: estimate $=0.09$, $\mathrm{p}<0.001 ; 3-6$ versus $12+$ : estimate $=0.11, p<0.001)$. The selected linear mixed model for BCVA variation is summarized in Table 3. The most important predictors of postoperative BCVA were the preoperative BCVA (estimate $=0.39, p<0.001$ ) and postoperative closure of the macular hole (estimate $=0.34, p<0.001$ ).

Postoperative BCVA was inversely associated with the duration of symptoms (estimate $=-0.78 \times 10^{-3}, p<0.001$ ) and the stage of hole at the time of operation (stage 2 versus 
stage 3: estimate $=-0.06, p=0.002$, stage 2 versus stage 4 : estimate $=-0.11, p<0.001)$. The role of ILM peeling on functional and anatomical results could not be evaluated, as most $(95.7 \%)$ surgeons peeled the ILM. The effect of incomplete versus complete tamponade on BCVA was not significant (estimate $=0.05, p=0.075$ ) and the effect of postoperative positioning on BCVA was negatively related to its duration (estimate $=-0.11 \times 10^{-1}, p=0.002$ ).

An improvement in BCVA was observed for combined vitrectomy and phacoemulsification (estimate $=0.10$, $\mathrm{p}<0.001$ ) as well as after cataract surgery regarding phakic eyes (estimate $=0.05, p=0.001$ ). The average number of $\log M A R$ lines gained after 12 months was $4.1 \pm 4.09$ lines for eyes undergoing combined surgery and $4.41 \pm 3.62$ for those who subsequently underwent cataract surgery and was not different $(p=0.168)$.

\section{Special Techniques}

The inverted ILM flap technique was used in a small sample of eyes: 238 eyes (5.6\%), predominantly in stage 4 macular holes (13\% of stage 4 macular holes were operated using ILM flap). Better anatomical and visual outcomes were noted regarding macular hole closures (odds ratio $=14.45, \mathrm{p}<0.001$ ) and BCVA (estimate $=1.42$ $\mathrm{x} 10^{-1}, \mathrm{p}=0.049$ ) when this technique was used compared to ILM peel in this subset of eyes.

\section{Complications}

Of 3085 initially phakic eyes, 1095 (35.5\%) underwent subsequent cataract surgery. Survival time analysis showed that the median time for cataract surgery was 8 months and that only $26.4 \%$ were operated within the first month following vitrectomy. Risk factors analysis confirmed that both the use of transconjunctival vitrectomy with trocars (odds ratio $=0.43, p=0.001$ ) and tamponades with lower gas concentrations (odds ratio $=1.18, p=0.023$ for concentration of gas $\times 10^{-1}$ ) were negatively associated with cataract surgery (Table 4). However, the use of C3F8 was more highly associated with cataract surgery than C2F6 (odds ratio $=2.02, p=0.038$ ), while there was no difference in the odds of cataract surgery comparing SF6 and C2F6 (odds ratio=1.42, $\mathrm{p}=0.277)$.

Regarding other complications, repeated surgery was performed in $24.3 \%$ of eyes with postoperative elevated edges, the results of which were not recorded. Retinal tears occurred in $5.1 \%$ of cases. The logistic mixed effects model for the probability of retinal tears indicated that use of trocars (odds ratio $=-1.246, p$ - value $=<0.001$ ) and combining surgery with cataract (odds ratio $=-0.688, \mathrm{p}$ value $=0.004$ ) were associated with a lower risk of retinal tears. Subsequent rhegmatogenous retinal detachment occurred in $3.6 \%$ of cases. Transient postoperative hypotony was observed in ten eyes, which was too low for statistical analysis. In these cases, no scleral sutures were used.

\section{DISCUSSION}

Surgery for macular hole is a commonly performed vitreoretinal operation with usually favorable outcomes [43]. Accordingly, there is a considerable clinical interest in the outcome of surgery, and the success rate for macular hole repair is often used as an indicator for evaluating vitreoretinal surgeons' performance. It is therefore important to present large outcome data from different surgeons to benchmark against, and to analyze the factors influencing the functional and anatomical outcomes for $\mathrm{MH}$ surgery. Herein, we presented the results of 4207 eyes with $\mathrm{MH}$ surgery from 140 surgeons, the largest comprehensive report to date.

While one recent retrospective study reported no difference in outcomes based on the duration of symptoms [44], this study confirmed the findings of earlier studies indicating that anatomical and functional successes are more often achieved in smaller macular holes with shorter durations and those with better preoperative visual acuity [45-47]. The duration of symptoms is a subjective parameter and should be interpreted cautiously. We also found that visual acuity improves during the first 12 months of surgery. This is also true for stage IV, chronic macular holes, and may be explained by restoration of foveal contour and the ellipsoid zone continuity [48].

We found that almost a half of patients underwent phacoemulsification during the study period. Better final BCVA was achieved in pseudophakic eyes and in those who underwent subsequent cataract surgery. However, in our study, timing of cataract surgery (simultaneous versus delayed) had no influence on the final 12-months outcome, which was previously observed in a small retrospective analysis [10].

One recent study showed that improvement with phacoemulsification after $\mathrm{MH}$ surgery is associated with better vision-related quality of life and recommended combined surgery [49]. Thus, it may be reasonable to schedule patients for phacoemulsification soon after vitrectomy for $\mathrm{MH}$ to offer a prompt improvement in vision.

Regarding tamponades, the type and dilution of gas did not significantly impact the anatomical or functional results. This in line with a recent meta-analysis that looked at the outcome of macular hole surgery operated with SF6 versus C3F8 gas [14], however, a large retrospective registry study indicated better vision in eyes operated 
with short acting gas [10]. Recent studies have also continued to debate postoperative positioning $[29,50$, 51], with one recent meta-analysis supported the use of face down positioning [28]. In this series, we observed worse functional results in patients who were advised longer periods of postoperative positioning. It might be because surgeons might have advised strict positioning in more complex cases. Thus, it may be reasonable to use shorter-acting gases such as SF6 and shorter positioning to reduce patient discomfort, improve adherence, and enable earlier airplane travel. However, as this study was not randomized, the data on positioning should be interpreted with caution.

Peeling of the ILM has been shown to be associated with improved anatomical success, however, its influence on visual success remains unclear. Data from a recent registry-based study from the New Zealand and Australia demonstrated that while peeling of the ILM increased the anatomical closure rate [10] this did not lead to better visual acuity outcomes at 3 or 12 months. In contrast, a meta-analysis of 4 randomized controlled studies conducted in 2014 demonstrated that ILM peeling was associated with a significant albeit small (approximately 1 LogMAR) visual improvement over no ILM peeling at 3 months [52]. In the present study, $95.7 \%$ of surgeons chose to peel the ILM, thus preventing a comparison. The use of staining for the purpose of ILM peeling was an important predictive factor of anatomical, but not functional success. The difference between the types of dyes was not statistically significant.

The inverted ILM flap technique was previously reported to increase the success rate in the treatment of large, stage 4 macular holes and macular holes associated with high myopia [9, 53, 54]. One recent retrospective study compared 300 eyes with ILM peel and 320 eyes with the inverted ILM flap technique and indicated a difference in overall success rates of $78.6 \%$ and $95.6 \%$, respectively in eyes with $\mathrm{MH} \geq 400$ [55]. We confirm the results of these studies, showing that this technique improves anatomical and functional results in large macular holes.

We found a $5 \%$ intraoperative rate of iatrogenic retinal tears and risk was lower in transconjunctival vitrectomy with the use of trocars. The reported rates of retinal tears with $\mathrm{MH}$ surgery in the literature vary widely with some studies reporting figures as high as 16\% [56]. Our results are comparable to those of a large national database study from the United Kingdom that reported retinal tears in $7 \%$ with PPV for $\mathrm{MH}$.

The results of our study need to be interpreted with caution because of the risk of bias associated with nonrandomization and its retrospective design. Similar to other database studies, there were missing data in our studies which may affect the study quality and there was a variation in techniques used based on surgeons' preference. Considering that a large number of physicians for more than 20 countries participated in this study, we expect the effect of these biases to be small. The large scale of data also makes it representative of retinal physicians' practice and suitable for surgeon benchmarking, as compared to studies originating from selected institutions.

\section{CONCLUSION}

This multicenter survey confirms earlier data from smaller studies which showed better results in low-stage, shortterm macular holes with good initial visual acuity. Phacoemulsification, performed before, during or after vitrectomy was one of the most important factors influencing functional results. In stage IV macular holes, the inverted ILM flap technique was associated with greater anatomical and functional success. Internal limiting membrane staining improved anatomical results without a significant difference between dyes. Moreover, longer positioning and use of long-acting gases or silicone oil were not shown to improve outcomes. A prospective study might add more insight on the effect of tamponade type and postoperative positioning on macular hole closure.

\section{ETHICAL DECLARATIONS}

Ethical Approval: An informed consent was obtained from all individual participants included in the study. The study follows the Tenets of the Declaration of Helsinki. The EVRS Committees approved the study design and ethical aspects of the investigation.

Conflict of Interest: None.

\section{FUNDING}

Oertli Instrumente AG and Maclodio Lions Club of Brescia, Italy supported the study with a financial grant. The funders had no role in study design, data collection and analysis, decision to publish, or preparation of the manuscript.

\section{ACKNOWLEDGMENTS}

Anja Leppich, the EVRS administrative director who led the data collection. Cristiano Varin, PhD (Ca' Foscari University, Venice, Italy), and Annamaria Guolo, PhD (University of Padova, Italy) for their help with the statistics. 


\section{REFERENCES}

1. McCannel CA, Ensminger JL, Diehl NN, Hodge DN. Population-based incidence of macular holes. Ophthalmology. 2009;116(7):1366-9. doi: 10.1016/j.ophtha.2009.01.052 pmid: 19576500

2. Stalmans $P$, Benz MS, Gandorfer A, Kampik A, Girach A, Pakola $S$, et al. Enzymatic vitreolysis with ocriplasmin for vitreomacular traction and macular holes. $\mathrm{N}$ Engl $\mathrm{J}$ Med. 2012;367(7):606-15. doi: 10.1056/NEJMoa1110823 pmid: 22894573

3. Neffendorf JE, Simpson ARH, Steel DHW, Desai R, McHugh $D A$, Pringle $E$, et al. Intravitreal gas for symptomatic vitreomacular adhesion: a synthesis of the literature. Acta Ophthalmol. 2018;96(7):685-91. doi: 10.1111/aos.13547 pmid: 28857483

4. Kelly NE, Wendel RT. Vitreous surgery for idiopathic macular holes. Results of a pilot study. Arch Ophthalmol. 1991;109(5):654-9. doi: 10.1001/archopht.1991.01080050068031 pmid: 2025167

5. Kim JW, Freeman WR, Azen SP, El-Haig W, Klein DJ, Bailey IL. Prospective Randomized Trial of Vitrectomy or Observation for Stage 2 Macular Holes. American J Ophthalmology. 1996;121(6):605-14. doi: 10.1016/s0002-9394(14)70625-7

6. Freeman WR, Azen SP, Kim JW, el-Haig W, Mishell DR, 3rd, Bailey I. Vitrectomy for the treatment of full-thickness stage 3 or 4 macular holes. Results of a multicentered randomized clinical trial. The Vitrectomy for Treatment of Macular Hole Study Group. Arch Ophthalmol. 1997;115(1):11-21. doi: 10.1001/archopht.1997.01100150013002 pmid: 9006420

7. Ando F, Sasano K, Ohba N, Hirose H, Yasui O. Anatomic and visual outcomes after indocyanine green-assisted peeling of the retinal internal limiting membrane in idiopathic macular hole surgery. Am J Ophthalmol. 2004;137(4):609-14. doi: 10.1016/j.ajo.2003.08.038 pmid: 15059697

8. Beutel J, Dahmen G, Ziegler A, Hoerauf H. Internal limiting membrane peeling with indocyanine green or trypan blue in macular hole surgery: a randomized trial. Arch Ophthalmol. 2007;125(3):326-32. doi: 10.1001/archopht.125.3.326 pmid: 17353402

9. Michalewska Z, Michalewski J, Adelman RA, Nawrocki J. Inverted internal limiting membrane flap technique for large macular holes. Ophthalmology. 2010;117(10):2018-25. doi: 10.1016/j.ophtha.2010.02.011 pmid: 20541263

10. Essex RW, Hunyor AP, Moreno-Betancur M, Yek JTO, Kingston ZS, Campbell WG, et al. The Visual Outcomes of Macular Hole Surgery: A Registry-Based Study by the Australian and New Zealand Society of Retinal Specialists. Ophthalmol Retina. 2018;2(11):1143-51. doi: 10.1016/j.oret.2018.04.022 pmid: 31047553

11. Michalewska Z, Michalewski J, Cisiecki S, Adelman R, Nawrocki J. Correlation between foveal structure and visual outcome following macular hole surgery: a spectral optical coherence tomography study. Graefes Arch Clin Exp Ophthalmol. 2008;246(6):823-30. doi: 10.1007/s00417-0070764-5 pmid: 18386040

12. Yamashita T, Sakamoto T, Terasaki H, Iwasaki M, Ogushi $Y$, Okamoto $F$, et al. Best surgical technique and outcomes for large macular holes: retrospective multicentre study in Japan. Acta Ophthalmol. 2018;96(8):e904-e10. doi: 10.1111/aos.13795 pmid: 29671948

13. Bainbridge J, Herbert E, Gregor Z. Macular holes: vitreoretinal relationships and surgical approaches. Eye (Lond). 2008;22(10):1301-9. doi: 10.1038/eye.2008.23 pmid: 18327161

14. Hecht I, Mimouni M, Blumenthal EZ, Barak Y. Sulfur Hexafluoride (SF6) versus Perfluoropropane (C3F8) in the Intraoperative Management of Macular Holes: A Systematic Review and Meta-Analysis. J Ophthalmol. 2019;2019:1820850. doi: 10.1155/2019/1820850 pmid: 30993017

15. Haritoglou C, Kampik A, Langhals H. II. Indocyanine Green Should Not Be Used to Facilitate Removal of the Internal Limiting Membrane in Macular Hole Surgery. Survey of Ophthalmology. 2009;54(1):138-41. doi: 10.1016/j.survophthal.2008.10.016

16. Ishida $M$, Ichikawa $Y$, Higashida $R$, Tsutsumi $Y$, Ishikawa $A$, Imamura Y. Retinal displacement toward optic disc after internal limiting membrane peeling for idiopathic macular hole. Am J Ophthalmol. 2014;157(5):971-7. doi 10.1016/j.ajo.2014.01.026 pmid: 24503409

17. Mester V, Kuhn F. Internal limiting membrane removal in the management of full-thickness macular holes. American J Ophthalmology. 2000;129(6):769-77. doi: 10.1016/s00029394(00)00358-5

18. Thompson JT, Haritoglu C, Kampik A, Langhals H. Should Indocyanine green should be used to facilitate removal of the internal limiting membrane in macular hole surgery. Surv Ophthalmol. 2009;54(1):135-8. doi: 10.1016/j.survophthal.2008.10.018 pmid: 19171215

19. Tognetto D, Grandin R, Sanguinetti G, Minutola D, Di Nicola $M$, Di Mascio $R$, et al. Internal limiting membrane removal during macular hole surgery: results of a multicenter retrospective study. Ophthalmology. 2006;113(8):1401-10. doi: 10.1016/j.ophtha.2006.02.061 pmid: 16877079

20. Vaziri K, Schwartz SG, Kishor KS, Fortun JA, Moshfeghi AA, Smiddy WE, et al. Rates of Reoperation and Retinal Detachment after Macular Hole Surgery. Ophthalmology. 2016;123(1):26-31. doi: 10.1016/j.ophtha.2015.09.015 pmid: 26505804

21. Kumagai K, Furukawa M, Ogino N, Uemura A, Larson E. Longterm outcomes of internal limiting membrane peeling with and without indocyanine green in macular hole surgery. Retina. 2006;26(6):613-7. doi: 10.1097/01.iae.0000236471.79066.fe pmid: 16829801

22. Conart JB, Selton J, Hubert I, Trechot F, El Adssi H, CreuzotGarcher $\mathrm{C}$, et al. Outcomes of macular hole surgery with short-duration positioning in highly myopic eyes: a casecontrol study. Ophthalmology. 2014;121(6):1263-8. doi: 10.1016/j.ophtha.2013.12.005 pmid: 24480709

23. Guillaubey A, Malvitte L, Lafontaine PO, Jay N, Hubert I, Bron A, et al. Comparison of face-down and seated position after idiopathic macular hole surgery: a randomized clinical trial. Am J Ophthalmol. 2008;146(1):128-34. doi: 10.1016/j.ajo.2008.02.029 pmid: 18440484 
24. Lange CA, Membrey L, Ahmad N, Wickham L, Maclaren RE, Solebo $\mathrm{L}$, et al. Pilot randomised controlled trial of face-down positioning following macular hole surgery. Eye (Lond). 2012;26(2):272-7. doi: 10.1038/eye.2011.221 pmid: 21941361

25. Mulhern MG, Cullinane A, Cleary PE. Visual and anatomical success with short-term macular tamponade and autologous platelet concentrate. Graefes Arch Clin Exp Ophthalmol. 2000;238(7):577-83. doi: 10.1007/s004170000154 pmid: 10955659

26. Tornambe PE, Poliner LS, Grote K. Macular hole surgery without face-down positioning. A pilot study. Retina. 1997;17(3):179-85. doi: 10.1097/00006982-19970500000001 pmid: 9196926

27. Tranos PG, Peter NM, Nath $R$, Singh $M$, Dimitrakos $S$, Charteris $D$, et al. Macular hole surgery without prone positioning. Eye (Lond). 2007;21(6):802-6. doi: 10.1038/sj.eye.6702339 pmid: 16575410

28. Xia S, Zhao XY, Wang EQ, Chen YX. Comparison of face-down posturing with nonsupine posturing after macular hole surgery: a meta-analysis. BMC Ophthalmol. 2019;19(1):34. doi: 10.1186/s12886-019-1047-8 pmid: 30691441

29. Lindtjorn B, Krohn J, Austeng D, Fossen K, Varhaug P, Basit S, et al. Nonsupine Positioning after Macular Hole Surgery: $A$ Prospective Multicenter Study. Ophthalmol Retina. 2019;3(5):388-92. doi: 10.1016/j.oret.2018.12.006 pmid: 31044728

30. Gupta D. Face-down posturing after macular hole surgery: a review. Retina. 2009;29(4):430-43. doi: 10.1097/IAE.0b013e3181a0bd01 pmid: 19359978

31. Tatham A, Banerjee S. Face-down posturing after macular hole surgery: a meta-analysis. $\mathrm{Br} J$ Ophthalmol. 2010;94(5):626-31. doi: 10.1136/bjo.2009.163741 pmid: 19767335

32. Adelman RA, Parnes AJ, Michalewska Z, Parolini B, Boscher C, Ducournau D, et al. Corrigendum to "Strategy for the Management of Diabetic Macular Edema: The European Vitreo-Retinal Society Macular Edema Study". Biomed Res Int. 2015;2015:694902. doi: 10.1155/2015/694902 pmid: 26798639

33. Adelman RA, Parnes AJ, Bopp S, Saad Othman I, Ducournau D, Evrs Macular Edema Study G. Corrigendum to "Strategy for the Management of Macular Edema in Retinal Vein Occlusion: The European VitreoRetinal Society Macular Edema Study". Biomed Res Int. 2015;2015:694690. doi: 10.1155/2015/694690 pmid: 26697492

34. Adelman RA, Parnes AJ, Ducournau D, European VitreoRetinal Society Retinal Detachment Study G. Strategy for the management of uncomplicated retinal detachments: the European vitreo-retinal society retinal detachment study report 1. Ophthalmology. 2013;120(9):1804-8. doi: 10.1016/j.ophtha.2013.01.070 pmid: 23601799

35. Adelman RA, Parnes AJ, Michalewska Z, Ducournau D, European Vitreo-Retinal Society Retinal Detachment Study G. Clinical variables associated with failure of retinal detachment repair: the European vitreo-retinal society retinal detachment study report number 4. Ophthalmology.
2014;121(9):1715-9. doi: 10.1016/j.ophtha.2014.03.012 pmid: 24766870

36. Adelman RA, Parnes AJ, Sipperley JO, Ducournau D, European Vitreo-Retinal Society Retinal Detachment Study G. Strategy for the management of complex retinal detachments: the European vitreo-retinal society retinal detachment study report 2. Ophthalmology. 2013;120(9):1809-13. doi: 10.1016/j.ophtha.2013.01.056 pmid: 23601805

37. Michalewska Z, Ducournau D, Adelman RA, Group ERS. How do vitrectomy parameters influence the results of rhegmatogenous retinal detachments repair? EVRS RD Study No. 3. Acta Ophthalmol. 2014;92(5):e416-7. doi: 10.1111/aos.12318 pmid: 24314312

38. Diggle P, Diggle PJ, Heagerty P, Liang K-Y, Heagerty PJ, Zeger S. Analysis of longitudinal data: Oxford University Press; 2002.

39. R Development Core Team R. R: A language and environment for statistical computing. $R$ foundation for statistical computing Vienna, Austria; 2015.

40. Pinheiro J, Bates D, DebRoy S, Sarkar D, Team RC. Linear and nonlinear mixed effects models. $\mathrm{R}$ package version. 2015;3(57):1-122.

41. Brostrom G. glmmML: Generalized linear models with clustering. R package version 10. 2013.

42. Akaike $H$. A new look at the statistical model identification. IEEE Transactions on Automatic Control. 1974;19(6):716-23. doi: 10.1109/tac.1974.1100705

43. Jackson TL, Donachie PH, Sparrow JM, Johnston RL. United Kingdom National Ophthalmology Database Study of Vitreoretinal Surgery: report 1; case mix, complications, and cataract. Eye (Lond). 2013;27(5):644-51. doi: 10.1038/eye.2013.12 pmid: 23449509

44. Stene-Johansen I, Bragadottir R, Petrovski BE, Petrovski G. Macular Hole Surgery Using Gas Tamponade-An Outcome from the Oslo Retrospective Cross-Sectional Study. J Clin Med. 2019;8(5). doi: 10.3390/jcm8050704 pmid: 31108955

45. Mester $U$, Becker $M$. [Prognostic factors in surgery of macular holes]. Ophthalmologe. 1998;95(3):158-62. doi: 10.1007/s003470050255 pmid: 9578693

46. Ullrich S, Haritoglou C, Gass C, Schaumberger M, Ulbig MW, Kampik A. Macular hole size as a prognostic factor in macular hole surgery. $\mathrm{Br} J$ Ophthalmol. 2002;86(4):390-3. doi: 10.1136/bjo.86.4.390 pmid: 11914205

47. Zou J, Zeng J. The macular microstructure repair and predictive factors of surgical outcomes after vitrectomy for idiopathic macular hole. Inter J Ophthalmology. 2019;12(5). doi: 10.18240/ijo.2019.05.25

48. Michalewska Z, Michalewski J, Nawrocki J. Continuous changes in macular morphology after macular hole closure visualized with spectral optical coherence tomography. Graefes Arch Clin Exp Ophthalmol. 2010;248(9):1249-55. doi: 10.1007/s00417-010-1370-5 pmid: 20379735

49. Wang Y, Liang X, Gao M, Liu J, Liu L, Liu W. Vision-related quality of life after pars plana vitrectomy with or without combined cataract surgery for idiopathic macular hole 
patients. Int Ophthalmol. 2019;39(12):2775-83. doi: 10.1007/s10792-019-01124-6 pmid: 31144239

50. Elborgy E, Starr M, Kotowski J, Chehade J, lezzi R. No Facedown Positioning Surgery for the Repair of Chronic Idiopathic Macular Holes. Retina. $2018 . \quad$ doi: 10.1097/iae.0000000000002396

51. Feist RM, Jr., Pomerleau DL, Feist R, Albert MA, Emond TL, Mason JO, 3rd, et al. Nonsupine positioning is preferred by patients over face-down positioning and provides an equivalent closure rate in 25- and 23-gauge macular hole surgery. Retin Cases Brief Rep. 2014;8(3):205-8. doi: 10.1097/ICB.0000000000000043 pmid: 25372439

52. Spiteri Cornish K, Lois N, Scott NW, Burr J, Cook J, Boachie C, et al. Vitrectomy with internal limiting membrane peeling versus no peeling for idiopathic full-thickness macular hole. Ophthalmology. 2014;121(3):649-55. doi: 10.1016/j.ophtha.2013.10.020 pmid: 24314837

53. Kuriyama S, Hayashi $H$, Jingami $Y$, Kuramoto N, Akita J, Matsumoto M. Efficacy of inverted internal limiting membrane flap technique for the treatment of macular hole in high myopia. Am J Ophthalmol. 2013;156(1):125-31 e1. doi: 10.1016/j.ajo.2013.02.014 pmid: 23622567

54. Michalewska Z, Michalewski J, Dulczewska-Cichecka K, Nawrocki J. Inverted internal limiting membrane flap technique for surgical repair of myopic macular holes. Retina. 2014;34(4):664-9. doi: 10.1097/IAE.0000000000000042 pmid: 24263468

55. Rizzo S, Tartaro R, Barca F, Caporossi T, Bacherini D, Giansanti F. Internal Limiting Membrane Peeling Versus Inverted Flap Technique for Treatment of Full-Thickness Macular Holes: A Comparative Study in a Large Series of Patients. Retina. 2018;38 Suppl 1:S73-S8. doi: 10.1097/IAE.0000000000001985 pmid: 29232338

56. Dogramaci M, Lee EJ, Williamson TH. The incidence and the risk factors for iatrogenic retinal breaks during pars plana vitrectomy. Eye (Lond). 2012;26(5):718-22. doi: 10.1038/eye. 2012.18 pmid: 22344186 